\title{
Nonlinear Compensation Using Digital Back-Propagation in Few-Mode Fibre Spans with Intermediate Coupling
}

\author{
Filipe M. Ferreira, Christian S. Costa, Stylianos Sygletos, Andrew D. Ellis \\ Aston Institute of Photonic Technologies, Aston University, Birmingham, UK (f.ferreira@aston.ac.uk)
}

\begin{abstract}
We investigate for the first time the performance of virtual back-propagation using multimode Manakov equations, derived for the weak- and strong-coupling regimes, after forward-propagation using a fully stochastic model over all linear coupling regimes.
\end{abstract}

\section{Introduction}

Mode-division multiplexing (MDM) over fewmode fibres (FMFs) has been proposed as a next-generation solution to overcome the impeding capacity exhaustion of current singlemode fibres (SMFs). To maximise the capacity of a FMF, additional impairments must be mitigated, namely: linear mode coupling (XT) ${ }^{1}$, differential mode delay $(\mathrm{DMD})^{1}$ and intermodal nonlinear effects $^{2,3}$. DMD and XT have been successfully mitigated using multi-input multi-output (MIMO) equalization after transmission over thousands of $\mathrm{kms}^{2}$. However, to the best of our knowledge, the applicability of nonlinear compensation techniques such as digital back-propagation (DBP) to MDM has not been studied yet, particularly for the most practical intermediate coupling regime.

In this paper, we evaluate DBP for MDM. Our DBP exploits multimode Manakov equations derived for either the weak- or strong-coupling regimes. The dependence of the compensation gain for the two DBP systems on mode coupling and DMD is analysed, showing that significant performance gain may be achieved.

\section{Nonlinear FMF Model}

The generalized nonlinear Schrödinger equation (GNLSE) for FMFs can be written as"

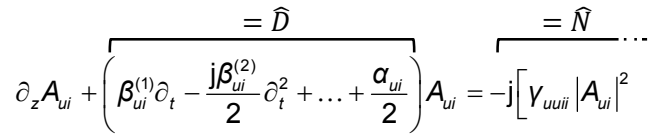

$$
\begin{aligned}
& \left.+2 \sum_{v \neq u} V_{u v i i}\left|A_{v i}\right|^{2}+\frac{2}{3} \sum_{v} V_{u v i j}\left|A_{v j}\right|^{2}\right] A_{\mu i}-\mathrm{j} \sum_{v k} C_{u v i k} A_{v k} e^{\mathrm{j}\left(\beta_{u i}^{(0)}-\beta_{v k}^{(0)}\right) z}
\end{aligned}
$$

where $i$ and $j$ are the orthogonal states of polarization of each mode $u$. $A_{u i}(z, t), \beta_{u i}{ }^{(1)}, \beta_{u i}{ }^{(2)}$ and $\alpha_{u i}$ are the slowly varying field envelope, group delay, group delay dispersion and attenuation, respectively. Yuvij is the nonlinear coefficient between $u i$ and $v j$, which depends on the intermodal effective area as shown in ${ }^{4}$.

In Eq. (1), $\widehat{D}$ is the differential operator that accounts for dispersion and attenuation, and $\widehat{N}$ is the nonlinear operator that accounts for all the intramodal and intermodal nonlinear effects ${ }^{4}$. The last term on the right-hand side accounts for the linear mode coupling arising from fibre structure

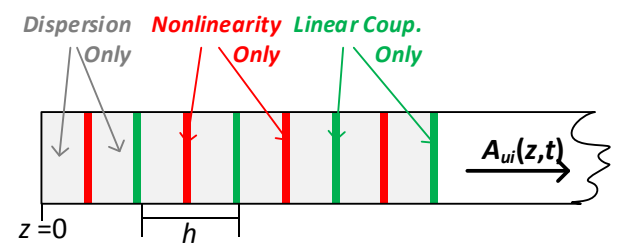

Fig. 1: Schematic illustration of the symmetric SSFM used for numerical simulations.

imperfections, where $C_{u v i j}$ are the coupling coefficients as derived in ${ }^{4}$.

For SMF the split-step Fourier method (SSFM) obtains an approximate solution of the Schrödinger equation by assuming that over a small distance $h$ the dispersive and nonlinear effects act independently. For FMFs, we extend such an approach by assuming that the mode coupling also acts independently. Such approximations require $h$ to be much shorter than the dispersion length $T_{0}^{2} /\left|\beta_{u}{ }^{(2)}\right|$ and the walk-off length $T_{0} /\left|\beta_{u^{(1)}}-\beta_{v}{ }^{(1)}\right|$ where $T_{0}$ is the pulse width, and shorter than the correlation length $L_{c}$ defined $^{3}$ for $X T\left(L_{c}\right)=\left[e^{2}-1\right] /\left[e^{2}+1\right]$.

Fig. 1 presents a schematic illustration of the symmetric SSFM used for numerical simulations. In a symmetric SSFM, the effect of nonlinearity is included in the middle of the segment rather than at the segment boundary ${ }^{5}$, providing higher accuracy. Finally, the step-size was selected by bounding the local error ${ }^{5}$, more computationally efficient at high accuracy than the other methods, e.g. nonlinear phase rotation.

The linear mode coupling is modelled considering fibre sections with a random displacement of the core centre position ${ }^{4}$. The coupling is set by varying the amplitude of the variation in lateral section offset as explained $\mathrm{in}^{3}$.

\section{Fibre Characteristics}

The fibre considered guides six linearly polarized (LP) modes: $\mathrm{LP}_{01}, \mathrm{LP}_{02}, \mathrm{LP}_{11 \mathrm{a}}, \mathrm{LP}_{11 \mathrm{~b}}, \mathrm{LP}_{21 \mathrm{a}}$ and $L_{21 b}$, the same fibre considered $i^{3}$. Tab. 1 shows the fibre linear characteristics at $1550 \mathrm{~nm}$, and Tab. 2 shows the intermodal nonlinear coefficients. In Tab. 1 can be seen that the FMF considered presents a DMD of $5.19 \mathrm{ps} / \mathrm{km}$. In Tab. 2, the uncoupled nonlinear coefficients are shown, whilst the uncoupled degeneracy factors 
Tab. 1: Fibre Linear Characteristics at 1550nm

\begin{tabular}{|l|r|r|r|r|r|r|}
\hline & \multicolumn{1}{|c|}{$\mathrm{LP}_{01}$} & \multicolumn{1}{|c|}{$\mathrm{LP}_{02}$} & $\mathrm{LP}_{11 \mathrm{a}}$ & $\mathrm{LP}_{11 \mathrm{~b}}$ & $\mathrm{LP}_{21 \mathrm{a}}$ & $\mathrm{LP}_{21 \mathrm{~b}}$ \\
\hline$G D[\mathrm{ps} / \mathrm{km}]$ & -0.29 & -2.93 & -0.66 & -0.66 & 2.27 & 2.27 \\
\hline$D[\mathrm{ps} /(\mathrm{nm} . \mathrm{km})]$ & 22.18 & 21.55 & 22.15 & 22.15 & 21.84 & 21.84 \\
\hline$S\left[\mathrm{fs} /\left(\mathrm{nm}^{2} \cdot \mathrm{km}\right)\right]$ & 66.45 & 61.46 & 66.15 & 66.15 & 63.68 & 63.68 \\
\hline$\alpha[\mathrm{dB} / \mathrm{km}]$ & 0.20 & 0.20 & 0.20 & 0.20 & 0.20 & 0.20 \\
\hline
\end{tabular}

Tab. 2: Nonlinear Coefficients $(\gamma u v)$ at 1550nm $\left[\mathrm{W}^{-1} / \mathrm{km}\right]$

\begin{tabular}{|l|c|c|r|r|r|r|}
\hline$u^{v}$ & $\mathrm{LP}_{01}$ & $\mathrm{LP}_{02}$ & $\mathrm{LP}_{11 \mathrm{a}}$ & $\mathrm{LP}_{11 \mathrm{~b}}$ & $\mathrm{LP}_{21 \mathrm{a}}$ & $\mathrm{LP}_{21 \mathrm{~b}}$ \\
\hline $\mathrm{LP}_{01}$ & 0.72 & 0.36 & 0.36 & 0.36 & 0.18 & 0.18 \\
\hline $\mathrm{LP}_{02}$ & 0.36 & 0.36 & 0.18 & 0.18 & 0.18 & 0.18 \\
\hline $\mathrm{LP}_{11 \mathrm{a}}$ & 0.36 & 0.18 & 0.55 & 0.55 & 0.27 & 0.27 \\
\hline $\mathrm{LP}_{11 \mathrm{~b}}$ & 0.36 & 0.18 & 0.55 & 0.55 & 0.27 & 0.27 \\
\hline $\mathrm{LP}_{21 \mathrm{a}}$ & 0.18 & 0.18 & 0.27 & 0.27 & 0.41 & 0.41 \\
\hline $\mathrm{LP}_{21 \mathrm{~b}}$ & 0.18 & 0.18 & 0.27 & 0.27 & 0.41 & 0.41 \\
\hline
\end{tabular}

can be find in Eq. (1). However, in the presence of mode coupling, it has been shown that the nonlinear distortion can be modelled using averaged coefficients, and new Manakov equations were derived for $\mathrm{FMFs}^{6,7}$. In the weak-coupling (WC) regime ${ }^{7}$, only the averaging over birefringence fluctuations must be considered, reducing the intramodal degeneracy factor to $8 / 9$ and the intermodal degeneracy factor to $4 / 3$. In the strong coupling (SC) regime $e^{6,7}$, the averaging includes all propagation modes. For $\mathrm{N}$-modes, the nonlinear operators for WC and SC are, respectively ${ }^{6,7}$ :

$$
\begin{aligned}
& \hat{N}=-j\left[\frac{8}{9} \sum_{k=\{i, j\}} Y_{u u i k}\left|A_{u k}\right|^{2}+\frac{4}{3} \sum_{\substack{v \neq u \\
k=\{i, j\}}} \gamma_{u v i k}\left|A_{v k}\right|^{2}\right] \\
& \hat{N}=-j \sum_{k=\{i, j\}} \kappa\left|A_{v k}\right|^{2}, \quad K=\frac{4}{3} \frac{2 N}{2 N+1}\left(\frac{1}{N^{2}} \sum_{\substack{u, V \\
k, V=\{i, j\}}} \gamma_{u v k k}\right)
\end{aligned}
$$

\section{Simulation Setup}

Twelve 28 GBd QPSK signals were transmitted, one per orthogonal polarization mode yielding a total bit rate of $672 \mathrm{~Gb} / \mathrm{s}$ per wavelength. Together with the information data, a preamble was transmitted consisting of constant amplitude zero autocorrelation (CAZAC) sequences, used for time synchronization and channel estimation. Root raised cosine filters with a roll-off factor of 0.001 were used for pulse shaping. Simulations considered $2^{16}$ symbols per polarization mode, from which $2^{11}$ were CAZAC symbols.

The receiver scheme is shown in Fig. 2. After homodyne detection, the baseband electrical signals were sampled at $56 \mathrm{GS} / \mathrm{s}$, yielding 12 digital signals at 2 samples/symbol. DBP was then implemented by launching the coherently received signals into a virtual fibre with characteristics of opposite-sign values of those in the transmission channel, except that no mode coupling was considered. Back-propagation was implemented using the modified SSFM presented with a fixed step size and considering the nonlinear operators derived for WC and SC, Eq. (2) and (3), respectively. As a reference, for linear

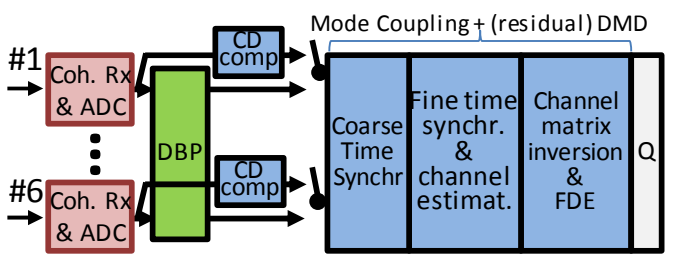

Fig. 2: Linear receiver and DBP scheme for 6 LP modes.

compensation, the coherently received signals were compensated for chromatic dispersion in the frequency domain using the values in Tab. 1.

In all cases, mode coupling and (residual) DMD were subsequently compensated for using data-aided channel estimation and equalization, as shown in Fig. 2. Coarse time synchronization was performed using the Schmidl \& Cox autocorrelation metric. Subsequently, fine-time synchronization and channel impulse response (CIR) estimation were performed by crosscorrelating with the training CAZAC sequences. The $12 \times 12 \mathrm{CIR}$ estimations were converted into the frequency domain. The MIMO frequency domain equalizer was calculated by inverting the channel matrix, and, finally, the Q-factor for each received signal was calculated using the mean and standard deviation of the received symbols.

\section{Results}

DBP performance was studied considering an optical super-channel consisting of 3 channels 28 Gbaud DP-QPSK per mode (spaced of 28.1 $\mathrm{GHz}$ ), giving a total bit rate of $2 \mathrm{~Tb} / \mathrm{s}$, over 10 spans of $100 \mathrm{~km}$. No sources of spontaneous noise were considered to assess the isolated impact of mode coupling on DBP performance. The fibre DMD was varied by scaling the mode group delay values in Tab. 1 . The $X T$ value was varied from -70 to $0 \mathrm{~dB} / 100 \mathrm{~m}$ covering the range of coupling values presented in the literature ${ }^{4}$. For forward propagation, the step size was selected by bounding the local error to be lower than $10^{-5}$, but for back-propagation a constant step size is used. Furthermore, the DBP performance was studied by back-propagating the 3 channels using either the WC-Manakov Eq. $(2)^{7}$ or the SC-Manakov Eq. (3) $)^{6,7}$. Finally, the Qfactor was averaged over the 12 polarization modes considering only the centre channels.

Fig. 3 shows the $Q$-factor gain over linear equaliser $(\Delta Q)$ for WC- and SC-DBP as a function of the DBP step after $1000 \mathrm{~km}$ for a DMD free fibre and launch power of $6 \mathrm{dBm} / \mathrm{ch}$. Note that by going from $200 \mathrm{~m}$ to $100 \mathrm{~m}$ negligible gain is observed. Thus, from this point, the step was kept at $100 \mathrm{~m}$ as complexity is out of the scope of this paper.

Fig. 4 shows the $Q$-factor as a function of the power per channel $\left(P_{c h}\right)$ after $1000 \mathrm{~km}$ for two DMD free fibres with crosstalk of -70 and $0 \mathrm{~dB} / 100 \mathrm{~m}$. First, it can be seen that WC- 


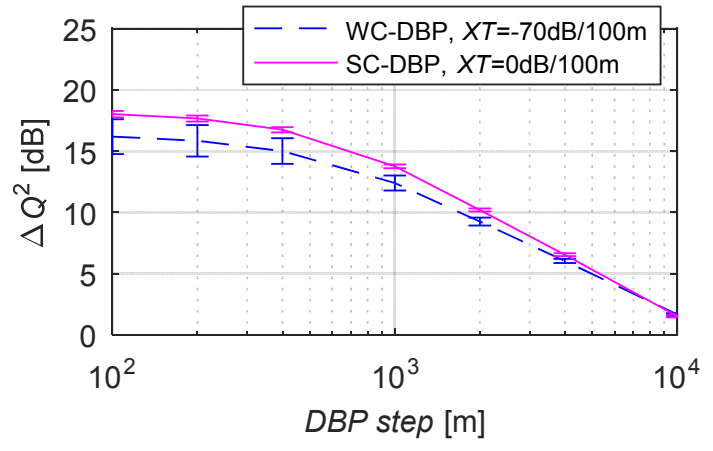

Fig. 3: $Q$-factor gain over linear eq. for WC- and SC-DBP as a function of the DBP step after $1000 \mathrm{~km}$ for $D M D=0$ and $6 \mathrm{dBm} / \mathrm{ch}$. Error bars for 10 repetitions.
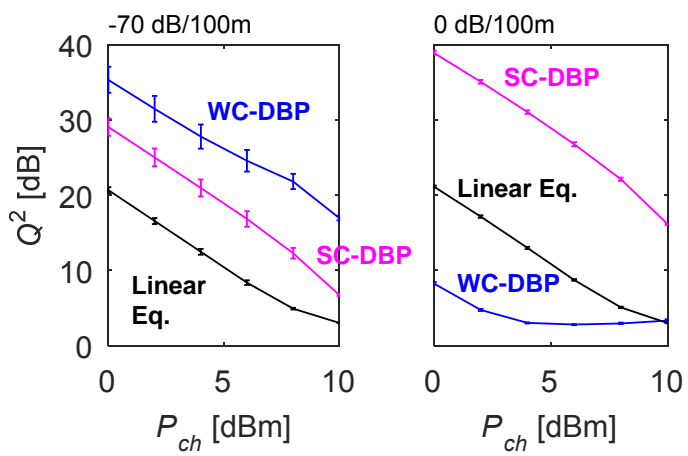

Fig. 4: $Q$-factor as a function of the power per channel after $1000 \mathrm{~km}$ for $X T=\{-70,0\} \mathrm{dB} / 100 \mathrm{~m}$ and for $D M D=0$. Error bars for 10 repetitions.

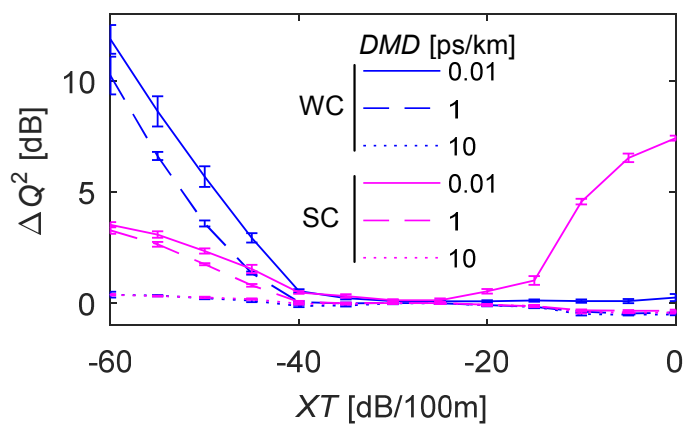

Fig. 5: $Q$-factor gain over linear eq. as a function of $X T$ after $1000 \mathrm{~km}$ for $D M D=\{0.01,1,10\} \mathrm{ps} / \mathrm{km}$ and $10 \mathrm{dBm} / \mathrm{ch}$. Error bars for 10 repetitions.

and SC-DBP provide their highest $Q$ improvement for low $X T$ and high $X T$ respectively, as expected. Moreover, one can observe that WC-DBP only provides gain for transmission over the weakly coupled fibre, while SC-DBP provides gain for both fibres. WC-DBP is particularly penalizing for high $X T$ values as the nonlinear coefficients in Eq. (2) are larger than the actual channel coefficients leading to large overcompensation. SC-DBP provides gain even for low $X T$ as the nonlinear coefficents in Eq. (3) are smaller than the actual channel coefficients leading to undercompensation.

Fig. 5 shows the $Q$-factor improvement over linear equalisation for WC- and SC-DBP as a function of $X T$ after $1000 \mathrm{~km}$ for three values of $D M D,\{0.01,1,10\} \mathrm{ps} / \mathrm{km}$ and at a launch power of $10 \mathrm{dBm} / \mathrm{ch}$. It can be seen that for negligible $D M D$ values $(<0.01 \mathrm{ps} / \mathrm{km})$, WC- and SC-DBP provide significant compensation in the regimes where their Manakov equations are valid (for $X T<-40 \mathrm{~dB} / 100 \mathrm{~m}$ and $X T>-20 \mathrm{~dB} / 100 \mathrm{~m}$, respectively). For small $D M D$ values $(\sim 1 \mathrm{ps} / \mathrm{km})$, WC-DBP still works within the WC regime $(X T<-40 \mathrm{~dB} / 100 \mathrm{~m})$, but SC-DBP provides no significant gain. SC-DBP using the uncoupled group delay values as in Tab. 1 no longer follows the actual $D M D$ evolution along the fibre thus incorrectly estimates the nonlinear distortion evolution. Furthermore, for moderate $D M D$ values $(\sim 10 \mathrm{ps} / \mathrm{km})$ neither of the DBP approaches work for any $X T$ value. This is because, for long distances $(1000 \mathrm{~km})$ and moderate $D M D$ values, even small $X T$ values lead to specific evolutions of the nonlinear and group delay operators, significantly different from that of the uncoupled operators in the Manakov approximation.

\section{Conclusions}

We have shown that significant nonlinear compensation is possible for WC FMF with small DMD. Compensation gains are maximised using the WC Manakov equation, but are also possible using the SC Manakov equation. Outside this region (SC or high DMD fibres) we observed no gain from a Manakov based DBP, and tracking of mode coupling will be required, as observed for SMF systems impacted by PMD. Whilst this paper has not focussed on DSP complexity, we anticipate that simplified algorithms which retain much of the benefit of Manakov DBP may be developed for WC low DMD fibres, or WC DMD managed links.

\section{Acknowledgements}

This work has been partially supported by the EU (619732-INSPACE, 654809-HSPACE and 659950INVENTION), and by EPSRC (EP/L000091/1-PEACE). To access the underlying data for this publication, see: http://researchdata.aston.ac.uk

\section{References}

[1] K. Ho, et al., "Statistics of group delays in multimode fiber with strong mode coupling," J. Lightw. Technol., 29(21), p 3119, 2011.

[2] R. Ryf et al., "Long-Distance Transmission over CoupledCore Multicore Fiber," ECOC, p. Th.3.C.3, 2016.

[3] F. Ferreira, et al., "Nonlinear Transmission Performance in Delay-Managed Few-Mode Fiber Links with Intermediate Coupling," OFC, p. Th2A.53, 2017.

[4] F. Ferreira, et al., "Nonlinear Semi-Analytical Model for Simulation of Few-Mode Fiber Transmission," Photon. Technol. Lett., 24(4), p.240, 2012.

[5] O. V. Sinkin, et al., "Optimization of the split-step Fourier method in modeling optical-fiber communications systems," J. Lightw. Technol., 21(1), p. 61, 2003.

[6] A. Mecozzi, et al., "Nonlinear propagation in multi-mode fibers in the strong coupling regime," Opt. Express, 20(11), p. 11673, 2012.

[7] S. Mumtaz, et al., "Nonlinear Propagation in Multimode and Multicore Fibers: Generalization of the Manakov Equations," J. Lightw. Technol., 31(3), p. 398, 2013. 